\title{
Análisis de los hábitos de estudio en dos grupos universitarios de microbiología alimentaria y su relación en el rendimiento académico por competencias
}

\section{Analysis of study habits in two university groups of food microbiology and their relationship to academic performance by competencies}

\author{
MEDINA-LERENA, Miriam Susana *†, COLÍN-MARTINEZ, Marco Antonio, PARADA- \\ BARRERA, Gloria y PÉREZ-RAMÍREZ, Miguel Ángel
}

Centro Universitario de Ciencias Biológicas y Agropecuarias (CUCBA), Universidad de Guadalajara, Camino Ing. Ramón Padilla Sánchez No. 2100, Predio Las Agujas, Nextipac, Zapopan, Jalisco, México. C. P. 4511.

ID 1 ${ }^{\mathrm{er}}$ Autor: Miriam Susana, Medina-Lerena / ORC ID: 0000-0003-4358-5400

ID $1^{\text {er }}$ Coautor: Marco Antonio, Martínez-Colín / ORC ID: 0000-0003-4905-0386

ID $2^{\text {do }}$ Coautor: Gloria, Parada-Barrera / ORC ID: 0000-0002-8432-1068

ID $3^{\text {er }}$ Coautor: Miguel Ángel, Pérez-Ramírez / ORC ID: 0000-0002-0405-9937

DOI: $10.35429 / J P D .2019 .7 .3 .30 .37$

Recibido 10 de Enero, 2019; Aceptado 25 de Marzo, 2019

\section{Resumen}

Los hábitos de estudio son métodos y estrategias que utiliza el estudiante para asimilar y adquirir un nuevo conocimiento y mejorar su rendimiento académico. El hábito requiere tres elementos importantes: el conocimiento, las capacidades y el deseo de mejorar, sin olvidar que los mismos requieren de esfuerzo, dedicación, disciplina y motivación. El objetivo del estudio fue conocer la relación entre los hábitos de estudio y el rendimiento académico en dos grupos universitarios de primer año en una competencia de Microbiología en Alimentos. Se utilizó un cuestionario para evaluar los hábitos de estudio de acuerdo a técnicas, organización, tiempo, distractores y eficiencia en el rendimiento académico. Los resultados demuestraron que existe una relación entre los hábitos de estudio y el bajo rendimiento académico. Esta información conyeva a tomar acciones de mejora en las técnicas y ambientes de aprendizaje que apoyen a corregir y resolver las deficiencias que se presenten en el quehacer diario del docente.

Hábitos de estudio, rendimiento académico, estudiantes universitarios

\begin{abstract}
Study habits are methods and strategies used by student to assimilate and acquire new knowledge and improve their academic performance. The habit requires three important elements knowledge, skills and the desire to improve, without forgetting that they require effort, dedication, discipline and motivation. The objective of the study was to know the relationship between study habits and academic performance in two first year university groups in a Food Microbiology competition. A questionnaire was used to evaluate study habits according to techniques, organization, time, distractor and efficiency in academic performance. The results showed that there is a relationship between study habits and poor academic performance. This information helps to take actions to improve learning techniques and environments that help correct and resolve the deficiencies that arise in the daily work of the teacher.
\end{abstract}

Study habits, academic performance, university student

Citación: MEDINA-LERENA, Miriam Susana, COLÍN-MARTINEZ, Marco Antonio, PARADA-BARRERA, Gloria y PÉREZ-RAMÍREZ, Miguel Ángel. Análisis de los hábitos de estudio en dos grupos universitarios de microbiología alimentaria y su relación en el rendimiento académico por competencias. Revista de Didáctica Práctica. 2019. 3-7: 30-37.

\footnotetext{
*Correspondencia al Autor (Correo Electrónico: miriam.mlerena @academicos.udg.mx)

$\dagger$ Investigador contribuyendo como primer autor.
} 


\section{Introducción}

Los comportamientos que una persona aprende por haberlos realizados de manera repetida se consideran hábitos. Existen los hábitos buenos y malos tanto en la salud, la alimentación y el estudio. Dentro de los hábitos buenos estos, contribuyen a que los personas consigan lograr sus objetivos y metas, siempre que estos sean trabajados en forma apropiada en el transcurso de su vida (Perrenod, 1996).

Dentro de los diferentes niveles de enseñanza existen numerosas variables que condicionan habitualmente el aprovechamiento académico en los estudiantes y su influencia sobre el conocimiento significativo. Por otro lado, la influencia que tienen los hábitos de estudio sobre los resultados escolares de los estudiantes. Sin embargo, no deben de confundirse los hábitos, referidos a prácticas invariables de las actividades mismas y las técnicas que son operaciones o recursos. El rendimiento académico es uno de los indicadores de calidad del sistema educativo. En conjunto conllevan a la eficacia del estudio.

Dentro de los desafíos más complicados y habituales a los que están expuestos los estudiantes en la educación en México son la deserción, el rezago estudiantil y bajos índices de eficiencia terminal. Donde se tienen como cifras generales con promedio nacional se alude que de cada 100 estudiantes que ingresan a la universidad, solamente entre 50 y 60 de ellos terminan de cursar todas sus asignaturas del plan de estudios cinco años después, de los cuales solo 20 se logran titular durante su primer año de egresados (ANUIES, 2001).

Mejorar el aprendizaje y el rendimiento académico de los estudiantes, especialmente en la educación superior, juega un papel esencial en el proceso de desarrollo de la sociedad. Por otro lado, la motivación en los estudiantes desarrolla un mejor entendimiento de los factores de aprendizaje; es posible que el contexto social diferente pueda ser uno de los elementos que influyan en la motivación y el auto-concepto académico (Isiksal, 2010).

Entre los hábitos de estudio que mejoran el desempeño académico de los estudiantes en educación superior destacan los siguientes:
La administración del tiempo; las habilidades cognitivas como la memoria, la atención y la concentración; la comprensión de lectura; los apuntes de clase; la redacción; el concepto de sí mismo; la motivación y voluntad; las relaciones interpersonales y el trabajo en equipo son factores deseables en un estudiante (Torres et al., 2009).

Mismos que los docentes buscan en los estudiantes a través de las tareas y actividades. Conocer los hábitos de cada estudiante ayudan a los docentes a que sus estudiantes conozcan las estrategias, las técnicas, las herramientas y los métodos que ellos aplican día a día para lograr cumplir en tiempo y forma con sus tareas, trabajos extra clases, exposiciones $\mathrm{y}$ exámenes.

Algunas investigaciones demuestran que los alumnos jóvenes optimistas cuentan con capacidades físicas e intelectuales y con hábitos de estudio bien cimentados en su vida estudiantil. Sin embargo, los bajos resultados demuestran que algunos hábitos de no atenderse en forma prioritaria por parte de autoridades y docentes, podrían provocar que los alumnos reprueben, fracasen y en el peor de los casos abandonen la escuela (Núñez y Sánchez, 1991).

\section{Justificación}

El incorrecto desarrollo de los hábitos de estudio comenzando con los niveles básicos de educación deriva en uno de los principales motivos por los que México tenga altos índices de fracaso académico. Este problema genera dificultades de aprendizaje que van más allá de lo estadístico y refleja deficiencias tanto en la calidad educativa que manifiestan los estudiantes de todos los niveles de estudio en el país (Tinto, 1992). Por lo que es importante que todo docente conozca y aprenda cuales son los canales de conocimiento en los estudiantes inscritos en su grupo. Mismos que los ayuden a mejorar sus estrategias y hábitos de estudio por los cuales adquiere el nuevo conocimiento de una manera más sencilla y al mismo tiempo sea motivador para mejorar la calidad educativa.

\section{Planteamiento del problema}

El interés por el hábito de estudio en los alumnos ha adquirido importancia debido a la gran demanda estudiantil y alta tasa de fracaso escolar. 
La realidad demuestra que no todos los estudiantes hacen frente con éxito a los nuevos desafíos que enfrenta debido al aumento de la exigencia, necesidad creciente de organización del trabajo académico, mayor dedicación al estudio, autonomía etc. Aunque las variables que condicionan el rendimiento académico son muy numerosas, se considera que una de las fundamentales que influyen en el bajo rendimiento escolar es el desconocimiento de un adecuado manejo de métodos y técnicas de estudio (Villegas, et. al., 2016).

La adquisición de competencias ayuda a desarrollar nuevas habilidades, capacidades y conocimientos que pueden ser utilizados en resolver problemas reales y situaciones concretas de su vida cotidiana en el quehacer académico; porque los continuos cambios que impone la sociedad moderna, obliga a que los estudiantes ser mejores profesionales. Si bien es cierto, que las acciones del maestro encaminadas a adquirir determinados conocimientos y habilidades que influyen en la vida del estudiante; toda persona en cierto nivel de la vida, empieza a desarrollar su reflexión y autonomía. Es el caso de estudiantes universitarios, donde ya han adquirido cierto grado de madurez que les permite reflexionar sobre los diversos aspectos de la vida, buscan siempre la manera de adquirir buenas notas que los lleven a obtener los mejores promedios al final de su carrera universitaria.

No obstante, en la experiencia del docente, se puede observar los diferentes estilos de estudio, pero no conoce los hábitos de cada estudiante para adquirir los conocimientos. Situación que pone en evidencia las deficiencias del estudiante que continua con el memorismo y muchas de las veces incentivándose la repetición de contenidos; por otro lado, algunos de los docentes mantienen el uso de metodologías tradicionales que impiden la formación de habilidades creativas e innovadoras obteniendo como resultados rendimientos muy bajos a lo que se suma la falta de hábitos de estudio en los estudiantes. Ya que el bajo rendimiento surge debido a que el alumno no sabe estudiar puesto que no organiza sus actividades ni posee métodos de trabajo ni técnicas de estudio adecuados que permita su aprendizaje significativo.
De aquí que se buscó y analizó si los hábitos de estudio utilizados en la competencia de Microbiología en Alimentos proporcionan elementos que apoyan el desarrollo de habilidades y competencias en el estudiante. De esta manera poder reconocer si los hábitos de estudio utilizados por los estudiantes mostraban relación con el rendimiento académico del mismo.

\section{Hipótesis}

Los hábitos de estudio utilizados por los estudiantes evidenciarán su relación con el rendimiento académico en dos grupos de un curso de microbiología.

\section{Objetivo general}

Conocer la relación entre los hábitos de estudio y el rendimiento académico en dos grupos universitarios de primer año en una competencia de Microbiología en Alimentos.

\section{Objetivos particulares}

- Conocer los hábitos de estudio en los alumnos de dos grupos de primer año en una competencia de Microbiología en Alimentos.

- Evaluar el rendimiento académico de dos grupos de Microbiología en Alimentos.

- Evaluar si los hábitos de estudio tienen relación con el rendimiento académico de los estudiantes de la competencia de microbiología en Alimentos.

- Evaluar sí los hábitos de estudio dentro y fuera del aula proporcionan elementos para el desarrollo de habilidades en el estudiante.

- Determinar si los hábitos de estudio y las estrategias utilizadas provocan un efecto en la adquisición del aprendizaje significativo en el estudiante.

\section{Marco teórico}

El hábito de estudio requiere fuertes cantidades de esfuerzo, dedicación y disciplina. Pero también se alimenta de impulsos que pueden estar generados por expectativas y motivaciones del estudiante que desea aprender. 
Por ello es necesario entender que el proceso de aprendizaje es complejo y requiere de una adecuada planeación y organización del tiempo (Isiksal, 2010).

De acuerdo a Jones et. al, (1991), todo lo que se efectué con el fin de optimizar la educación derivará en resultados pobres en cuestión que se valla dejando por lado el importante papel que tiene el estudiante en el proceso de aprendizaje; a mayor medida cuando no se consideran aquellas habilidades que el estudiante posee para así lograr de manera exitosa su desempeño académico (Stanley et. al., 1999).

La exigencia del día a día de mejorar el estudio en los estudiantes es una constante pedagógica. Donde muchas de las propuestas para su calidad son elaboradas por los propios docentes y en base a su experiencia en su quehacer diario. Moal estudiante en determinadas estrategias de atención, elaboración y organización de la información, al tiempo que se favorece la meta cognición o regulación de los propios procesos de pensamiento y de aprendizaje.

Jara et al. (2008) indican que: "el rendimiento académico es la capacidad de respuesta que tiene un individuo a estímulos, objetivos y propósitos educativos previamente establecidos". Por otro lado, los autores Fernández y Rubal (2014) definen el rendimiento académico como "un indicador del nivel de aprendizaje alcanzado por el alumno". Un rendimiento académico bajo indica que el estudiante no ha adquirido de forma adecuada y completa los conocimientos, por lo que carece de técnicas y habilidades necesarias para la solución de problemas del curso. Mismos que se requieren en la vida real al buscar solucionar problemas a los cuales se va a enfrentar en la vida profesional (Jara et al., 2008; Fernández y Rubal 2014).

Los factores que se asocian a un bajo rendimiento académico han sido objeto de estudio son los hábitos de estudio. Indican que: el estudiante hace un mayor uso de estrategias de aprendizaje memorísticas, fallas en la capacidad de reflexión y de análisis crítico, escaso tiempo dedicado al estudio y la preparación se limita a un día antes del examen (Jara et al., 2008).
Según Contreras, et. al., (2008) el bajo rendimiento académico se debe analizar desde una perspectiva multi-causal que aborde de manera integral los factores ligados al comportamiento del estudiante, su personalidad, naturaleza afectiva entre otros. Indican que se debería abordar desde tres perspectivas: personal, familiar y social. Relacionados con el comportamiento de los hábitos de estudio del estudiante (Contreras, et. al., 2008).

El interés sobre el desarrollo de la psicología cognitiva se ha ido incrementado por los aportes en el campo educativo, a través del aprendizaje significativo y estratégico, en los procesos de formación, en los distintos niveles educativos (Villamizar, 2008).

\section{Material y métodos}

El presente estudio se realizó con dos grupos de la Licenciatura de Alimentos de la Universidad de Guadalajara. El grupo A T/M se conformó con la población de 19 alumnos y el grupo B T/V con una población de 15 alumnos, ambos inscritos en el curso de la competencia de microbiología en alimentos durante el ciclo 2018-B. Con edades de entre los 18 a 22 años, hombres y mujeres. Se aplicó un estudio de tipo diagnóstico-cuantitativo con el propósito de conocer si los hábitos de estudio y el rendimiento académico tenían relación en el desempeño académico de los estudiantes. Con el fin de obtener resultados que pudieran servir para buscar las mejores estrategias para adquirir el conocimiento y al mismo tiempo mejorar el proceso de enseñanza-aprendizaje en el curso de microbiología de los alimentos que se imparte en la carrera de Licenciatura en Alimentos.

Se realizó un cuestionario, para obtener la información y conocer la opinión de cada uno sobre los hábitos de estudio utilizados. Con el fin de conocer si estos eran propicios para poder adquirir el conocimiento de la competencia. Para obtener los resultados y conocer el rendimiento académico se recabaron los datos de las evaluaciones finales de cada Sub-Unidad de competencia. Posteriormente se realizó la evaluación de cada una. Las cuales se menciona a continuación:

$\mathrm{T} 1=$ Identificar las fuentes $\mathrm{y}$ mecanismos de contaminación microbiana de los alimentos.

MEDINA-LERENA, Miriam Susana, COLÍN-MARTINEZ, Marco Antonio, PARADA-BARRERA, Gloria y PÉREZ-RAMÍREZ, Miguel Ángel. Análisis de los hábitos de estudio en dos grupos universitarios de microbiología alimentaria y su relación en el rendimiento académico por competencias. Revista de Didáctica Práctica. 2019 
$\mathrm{T} 2$ = Identificar y describir los factores que afectan el desarrollo de los microorganismos en el alimento y la forma en que pueden funcionar como barreras para su control.

$\mathrm{T} 3$ =Analizar e interpretar la presencia de microorganismos aditivos e indicadores en alimentos

$\mathrm{T} 4=$ Describir los principales microorganismos patógenos en alimentos y sus consecuencias en la salud humana.

Para los resultados de cada una de las Subunidades de la competencia se realizó un análisis de datos donde se corrió un ANOVA en el programa Excel@ para promediar y encontrar las diferencias significativas en las evaluaciones obtenidas de los diferentes grupos que formaron parte del estudio. En la que se evaluaron cada una de las subunidades de competencia del curso de microbiología de Alimentos.

\section{Resultados}

Se obtuvieron en este estudio los siguientes resultados, 34 Cuestionarios realizados a a todos los estudiantes activos de Microbiología de Alimentos. Hombres y mujeres con un promedio de edad de 21 años. Se promediaron los resultados de cada grupo de forma independiente. El promedio de los dos grupos se obtuvo de cada Subunidad de aprendizaje como se muestra en la siguiente tabla.

\begin{tabular}{|l|c|r|r|r|}
\hline \multicolumn{1}{c}{ Grupo } & \multicolumn{4}{c|}{ Sub-Unidades de } \\
& \multicolumn{4}{c|}{ aprendizaje } \\
& \multicolumn{1}{c}{ T1 } & T2 & T3 & T4 \\
\hline A Matutino & 80 & 83 & 85 & 88 \\
\hline B Vespertino & 77 & 81 & 83 & 86 \\
\hline
\end{tabular}

Tabla 1 Promedios de los tratamientos (T) en el rendimiento académico de los estudiantes de Microbiología de los Alimentos

Los resultados muestran que existen diferencias entre los promedios $(\mathrm{P} \leq 0.01)$. Para la separación de medias se corrió una prueba de Duncan al 5\%. El mejor promedio lo alcanzo el aprendizaje fue el T3, mientras que el T1 fue el peor de los tratamientos. En la encuesta realizada se encontró que el $97 \%$ de los alumnos estudiaba extra clase para entender mejor los conceptos, aclarar temas, mejorar sus conocimientos, sin embargo, el restante 3\% solo lo realizaba esporádicamente ya que no necesitaba estudiar.
Llamo la atención que la mayoría de los alumnos (98\%) dedica mayor tiempo cuando tienen examen y la razón principal fue para sacar buenas notas. Se les cuestiono sobre la mejor manera de estudiar y la mayoría $(96 \%)$ respondió que era leyendo, repasando los resúmenes tomados en clase, cabe destacar que la mayoría respondió que tiene la costumbre de memorizar los conceptos solo para examen.

Mientras que el 5\% comento que memorizar siempre es pasajero y tiende a olvidarse si no hacen algo práctico, para aplicar el conocimiento. Al preguntarles la clase de distractores tenían cuando estudiaban la mayoría (98\%) contesto que los aparatos electrónicos (en los cuales se incluían las conversaciones telefónicas, las redes sociales, el internet y la televisión). Mientras que el 2\% restante comento que las visitas inesperadas y los ruidos de la calle. Es importante decir que la mayoría de los estudiantes comento que prefiere estudiar en la noche ya que es mucho más tranquila que el día.

Los resultados revelaron que existe un alto porcentaje de estudiantes que mejoran sus notas al cambiar sus hábitos de estudio. Cuando existe una motivación por parte del docente se puede conseguir que los hábitos de estudio formen parte de la disciplina como un elemento básico para trabajar constantemente en la competencia asignada como demostraron los promedios de calificación en esta competencia al ser evaluados. Las buenas notas obtenidas por su esfuerzo y dedicación los motivaban a organizar y administrar el tiempo dedicado al estudio. Es importante señalar que se les cuestiono acerca de porque ellos estudiaban y la mayoría contesto que era porque quería superarse personalmente y profesionalmente, tener buenos ingresos, para aprender más e inclusive para tener un plan de vida, cosas que implican confianza en sí mismos.

\section{Discusión}

De acuerdo a los resultados obtenidos en este trabajo concuerdan con Jara et al. (2008) el cual mencionan que "las causas del bajo rendimiento de los estudiantes están relacionadas con la dificultad para el auto-aprendizaje, escasos conocimientos básicos sobre las ciencias; así como la constante práctica de un aprendizaje memorístico". 
Donde por tales motivos el bajo rendimiento académico del estudiante denota una situación que afecta a toda la comunidad estudiantil que de manera global encierra: estudiantes, padres, maestros y en consecuencia a toda la sociedad. Por otra parte, conviene que los alumnos tengan hábitos de estudio, así como el uso de técnicas adecuadas para sacar el máximo provecho al estudio, el cual implica la práctica bien ejecutada e intensiva de la energía empleada para tal como lo señala Pérez y Barberis (2005).

Los autores Martínez y Torres (2001) revelan que "No todos los estudiantes enfrentan con buen suceso en los desafíos de la vida universitaria que implica una mayor dedicación al estudio y mayor organización del trabajo académico", entre otros aspectos. Sin embargo, no hay que olvidar que el profesor debe de brindar un ambiente propicio y motivador donde los estudiantes puedan lograr el objetivo que es la apropiación del conocimiento por medio de una gama de estrategias y hábitos de estudio. Autores como Bajwa et. al. (2011), mencionan que un estudiante no puede usar habilidades de estudio eficaces, hasta que no tenga buenos hábitos de estudio y argumentan que un individuo aprende con mayor rapidez y profundidad. Además, reiteran que estudiar de forma eficaz y eficiente consiste más que la memorización de hechos, en saber dónde y cómo obtener la información importante y la capacidad de hacer uso inteligente de la misma.

Es necesario considerar siempre el hábito de estudio cuando se quiere avanzar en el aprendizaje. Por otra parte, conviene el uso de técnicas adecuadas para sacar el máximo provecho al estudio, el cual implica la practica bien ejecutada e intensiva de la energía empleada para tal (Pérez y Barberis 2005).

En un estudio realizado por Etxeberria, et. al. (2017) encontraron que en el análisis de las encuestas iniciales y finales realizadas al alumnado permitió detectar aquellas áreas temáticas que conviene profundice previamente. Además, se obtuvo información sobre las dificultades procedimentales observadas por el alumnado. Permitiendo diseñar contenidos teóricos y actividades prácticas que el alumnado pueda utilizar (Etxeberria, et. al. 2017).
De acuerdo con Friola y Velázquez (2011), debe de existir el interés y/o preocupación por parte del profesor para aplicar las estrategias didácticas adecuadas o pertinentes a sus grupos de estudiantes. Por esta razón concuerda con nuestro principal objetivo: el conocer los hábitos de estudio por los cuales el alumno aprende mejor dentro y fuera del aula fomenta la apropiación de saberes y en esencia la construcción del conocimiento. El cual se logra gracias al apoyo, guía y motivación por parte del docente en las diferentes actividades del contenido de las unidades de competencia del curso. Donde los estudiantes desarrollen más capacidades y habilidades y por ende estén más interesados en actividades extra clase e integren de manera apropiada la construcción del nuevo conocimiento. Los estudiantes denotan voluntad, responsabilidad e interés si tienen buenos hábitos de estudio y esto conlleva a obtener buenas notas lo que supera las expectativas del profesor. Como lo demuestra a la hora de desarrollar intervenciones preventivas para evitar la deserción universitaria, Garzón y Gil, 2017; sugieren privilegiar la mejora en las habilidades previamente descritas en el alumnado de nuevo ingreso, obviamente dentro de un modelo institucional integral y estratégico para el manejo de la retención de los estudiantes (Garzón y Gil, 2017).

\section{Conclusión}

Es necesario considerar siempre el hábito del estudio cuando se quiere avanzar en el aprendizaje. Por otra parte, debe considerarse que la motivación es un factor determínate y a la vez positivo por parte del docente al hacer conscientes a los estudiantes que valoren su colaboración en las actividades y tareas en las unidades de competencia cursadas para obtener buenas notas y por ende desarrollen su proceso de enseñanza-aprendizaje y al mismo tiempo trascienda en un aprendizaje significativo. En el sentido que constituye una vivencia satisfactoria de retroalimentación como de crecimiento personal y social.

Por último, el docente que dirige y guía a sus estudiantes debe de crear el interés para que puedan construir nuevas ideas, comunicar y confrontar sus ideas, con el fin de desarrollar las competencias a través de la interacción social como una parte esencial del constructivismo. 
Como toda investigación, ésta ha quedado inconclusa. Sin embargo, el objetivo de este estudio se cumplió, ya que se encontró que los hábitos de estudio tienen relación con el rendimiento académico del estudiante. Por lo que resulta importante señalar que constantemente se debe estar modificando y revisando los complementos de evaluación, para que los diseños de las actividades implementadas permitan evaluar el desarrollo de las habilidades y conocimientos que están adquiriendo los estudiantes universitarios.

\section{Referencias}

Asociación Nacional de Universidades e Instituciones de Educación Superior (ANUIES), (2001). Programas institucionales de tutoría. Una propuesta de la ANUIES para su organización y funcionamiento en las instituciones de Educación Superior. México. Ed. ANUIES.

Bajwa, N., Gujjar, A., Shaheen, G., y Ramzan, M. (2011). A comparative study of the study habits of the students from formal and nonformal systems of education in Pakistan. International Journal of Business \& Social Science, 2(14):175-186, http://www.scielo.org.mx/scielo.php?script=sci _nlinks\&ref $=5159708 \& \mathrm{pid}=$ S0185-

$2760201200030000300004 \& \operatorname{lng}=\mathrm{es}$

Contreras, K., Caballero, C., Palacio, J. y Pérez, A. M. (2008). Factores asociados al fracaso académico en estudiantes universitarios de Barranquilla (Colombia). Obtenido de Psicología desde el Caribe, Universidad del Norte. ISSN 0123-417X Psicología desde el Caribe, 22: 110-135 https://www.redalyc.org/pdf/213/21311866008. pdf

Etxeberria, P., Alberdi, E., Eguia, I y García M. J. (2017). Análisis del Rendimiento Académico en Relación al Perfil de Ingreso del Alumnado e Identificación de Carencias Formativas en Materias Básicas de dos Grados de Ingeniería. Formación Universitaria. 10(4), 67-74

Fernández, M., \& Rubal, N. (2014). Los hábitos de estudio y el rendimiento académico en el primer año de la carrera de medicina. Obtenido de Congreso Virtual de Ciencias Morfológicas.: http://www.morfovirtual2014.sld.cu/index.php/ Morfovirtual/2014/paper/download/198/2 8
Friola, P. y Velázquez, J. (2011). Estrategias didácticas por competencias: Diseños eficientes de intervención pedagógica para la educación básica, media superior y superior. (pp. 98). México: Centro de Investigación Educativa y Capacitación Institucional, S.C.

Hernández, Pedro y García, Luis. Psicología y enseñanza del estudio, Madrid, Pirámide, 1991. Garzón, A. y Gil, J. (2017). El papel de la procrastinación académica como factor de la deserción universitaria. Revista Complutense de Educación, 28 (1), 307-324.

Isiksal, M. (2010). A ComparativStudy on Undergraduate Students' Academic Motivation and Academic Self-Concept. The Spanish Journal of Psychology, http://redalyc.uaemex.mx/redalyc/ src/inicio/ArtPdfRed.jsp?iCve=17217376005\#, pp. 572-585, PMID 20977008

Jara, D., Velarde, H., Gordillo, G., Guerra, G., Arroyo, C., \& Figueroa, M. (2008). Factores influyentes en el rendimiento académico de estudiantes del primer año de medicina. An. Fac. Med. 69(3), Lima.: http://www.scielo.org.pe/scielo.php?pid=S1025 $-55832008000300009 \&$ script=sci_arttext

Jones, Craig H.; Slate, John R.; Bell, Stacet y Saddler, C. Douglas. (1991). Helping high school students improve their academic skills: a necessary role for teachers. The High School Journal, 198-202.

Martínez-Otero, V y Torres, L. (2001). Análisis de los hábitos de estudio en una muestra de estudiantes universitarios. Revista Iberoamericana de educación. 32 (2).

Núñez, C. y Sánchez, J. (1991). Hábitos de estudio y rendimiento en EGB y BUP. Un estudio comparativo. Revista Complutense de Educación, Vol. 2 (1). Universidad Complutense,

Madrid.http://revistas.ucm.es/index.php/RCED/ article/view/RCED9191130043A/18163

Pérez, V. M. O., \& Barberis, L. T. (2005). Análisis de los hábitos de estudio en una muestra de alumnos universitarios. Revista Iberoamericana de Educación, 36(7), 1-9. 
Perrenoud, P. (1996). La construcción del éxito y del fracaso escolar: hacia un análisis del éxito, del fracaso $\mathrm{y}$ de las desigualdades como realidades construidas por el sistema escolar. Madrid. ISBN 9788471123466. Ed. Morata.

Stanley, Bárbara; Slate, John R. y Jones, Craig H. (1999). Study behaviors of college preparatory and honors students in the ninth grade. The High School Journal, 165-171.

Tinto, V. (1992). El abandono en los estudios superiores. Una nueva perspectiva de las causas de abandono y su tratamiento. México. Ed. UNAM/ANUIES. Cuadernos de Planeación Universitaria, 2da época, 6(2).

Tirado, F., Martínez, M., Covarrubias, P., López, M., Quesada, R., Olmos, A., DíazTorres, M., Tolosa, I., Urrea, M., Monsalve, A. (2009). Hábitos de estudio vs fracaso académico. En Revista Educación de la Universidad de Costa Rica, 33(2). http://redalyc.uaemex.mx/pdf/440/4401205800 2.pdf

Villamizar, Acevedo, G., (2008) Relación entre las estrategias de aprendizaje y rendimiento académico en estudiantes de Psicología, Revista docencia Universitaria 6(9):71-94.

Villegas, C., Muñoz, F., \& Villegas, R. (2016). Hábitos de estudio de los alumnos en el área de Química Orgánica. Obtenido de Revista Biotecnia, 11(3) http://biotecnia.ojs.escire.net/index.php/biotecni a/article/viewFile/72/66 